\title{
Evaluation of endoscopic cytological diagnosis of unresectable pancreatic cancer prior to and after the introduction of endoscopic ultrasound-guided fine-needle aspiration
}

\author{
TOMOYUKI USHIJIMA ${ }^{1}$, YOSHINOBU OKABE ${ }^{1}$, YUSUKE ISHIDA $^{1}$, GEN SUGIYAMA ${ }^{1}$, YU SASAKI ${ }^{1}$, \\ KEI KURAOKA ${ }^{1}$, MAKIKO YASUMOTO ${ }^{1}$, TOMOKI TAIRA ${ }^{2}$, YOSHIKI NAITO ${ }^{3}$, \\ MASAMICHI NAKAYAMA ${ }^{3}$, OSAMU TSURUTA ${ }^{1}$ and MICHIO SATA ${ }^{1}$ \\ ${ }^{1}$ Division of Gastroenterology, Department of Medicine, Kurume University School of Medicine; \\ Departments of ${ }^{2}$ Diagnostic Pathology and ${ }^{3}$ Pathology, Kurume University School of Medicine, Kurume, Fukuoka, Japan
}

Received March 27, 2014; Accepted April 7, 2014

DOI: $10.3892 / \mathrm{mco} .2014 .277$

\begin{abstract}
With the advances in the multidisciplinary treatment of pancreatic cancer (PC) over the last few years, it is crucial to obtain a histopathological diagnosis prior to treatment. Histopathological diagnosis for unresectable PC is currently performed with endoscopic retrograde cholangiopancreatography (ERCP) in combination with endoscopic ultrasound-guided fine-needle aspiration (EUS-FNA). We retrospectively assessed the results of these two methods and investigated diagnostic performance according to the location of the lesion and the complications. This study was conducted on a series of 263 consecutive cases of unresectable PC diagnosed with endoscopic cytology. Up to 2006, ERCP-guided cytology (group A) was performed as the first choice for the diagnosis of PC. EUS-FNA was introduced in 2007 and became the first choice thereafter (group B), except in cases with obstructive jaundice, in which ERCPguided cytology during endoscopic biliary stenting (EBS) remains the first choice. There were statistically significant differences in the overall cancer-positive rate between groups A and B (60.4 vs. $75.3 \%, \mathrm{P}=0.01)$. The cancer-positive rate in the pancreatic body and tail was significantly higher in group B (59.5 vs. $83.3 \%, \mathrm{P}=0.005)$, whereas there were no significant differences regarding cancer of the pancreatic head. The complication rate was $4.95 \%$ in group A and $3.09 \%$ in group $B(P=0.448)$. The endoscopic cytology cancer-positive rate in unresectable $\mathrm{PC}$ cases was increased as a result of the introduction of EUS-FNA. In conclusion,
\end{abstract}

Correspondence to: Dr Yoshinobu Okabe, Division of Gastroenterology, Department of Medicine, Kurume University School of Medicine, 67 Asahi-machi, Kurume, Fukuoka 830-0011, Japan

E-mail: okabe_yoshinobu@kurume-u.ac.jp

Key words: unresectable pancreatic cancer, endoscopic cytology, endoscopic retrograde cholangiopancreatography, endoscopic ultrasound-guided fine-needle aspiration we recommend performing EUS-FNA in combination with ERCP-guided cytology in cases with a lesion in the pancreatic head that requires EBS.

\section{Introduction}

With the advances in the multidisciplinary treatment of pancreatic cancer (PC) over the last few years, it is crucial to obtain a histopathological diagnosis prior to treatment. With the introduction of endoscopic retrograde cholangiopancreatography (ERCP), ERCP-guided pancreatic juice and bile cytology and bile and pancreatic duct brush cytology or biopsy were adopted for the diagnosis of PC (1,2). Vilmann et al (3) subsequently developed endoscopic ultrasound-guided fine-needle aspiration (EUS-FNA), expanding the range of endoscopic collection methods for PC. As ERCP requires a highly skilled technique and, particularly, due to the risk of post-ERCP pancreatitis (PEP), EUS-FNA, which is considered to have a low incidence of complications, was widely adopted, primarily in Western countries. However, the use of EUS-FNA in Japan has been delayed, due to concerns regarding cancer seeding associated with EUS-FNA (4); therefore, the number of institutions performing EUS-FNA for pancreatic cystic lesions or PC that have been scheduled for resection is currently limited. Consequently, in Japan, endoscopic cytology or histological diagnosis for PC is currently performed with a combination of ERCP and EUS-FNA; however, the number of available studies is insufficient to clearly determine the optimal endoscopic collection method.

The purpose of this clinical study was to retrospectively evaluate the results of endoscopic cytology diagnosis in unresectable PC by the conventionally performed ERCP-guided collection method and after the introduction of EUS-FNA and to determine the usefulness of the two methods and the associated complications.

\section{Patients and methods}

Patients. A total of 263 consecutive patients with unresectable PC who underwent endoscopic cytology in our hospital 
between 2002 and 2012 were included in this study. Unresectability was confirmed in cases where a surgeon and a radiologist performed various diagnostic imaging examinations and diagnosing the case as Japan Pancreas Society classification stage $4 \mathrm{a}$ (major blood vessel invasion) or stage $4 \mathrm{~b}$ (distant metastases), cases in which surgery was not feasible due to the risk of general anesthesia because of cardiopulmonary disease and cases in which the patient was elderly and was considered to be unlikely to tolerate surgery. PC was definitively diagnosed based on a 6-month or longer course with radiological imaging, clinical symptoms and biochemical findings.

ERCP-guided pancreato-biliary brush cytology and EUSguided fine-needle aspiration. Prior to 2006, the endoscopic cell or tissue collection method for unresectable PCs in our hospital consisted of performing ERCP-guided pancreatic juice cytology (including pancreatic duct brush cytology) or bile duct cytology as the first choice. Since 2007, when EUS-FNA was introduced, ERCP-guided specimen collection is adopted as the first choice when endoscopic biliary stenting (EBS) is performed to relieve obstructive jaundice and EUS-FNA is adopted as the first choice (EUS-FNA 1st) in cases without obstructive jaundice. Additionally, in cases where an adequate specimen was not obtained by ERCP-guided collection, EUS-FNA may be subsequently performed (EUS-FNA 2nd) after obtaining the patient's consent.

In the ERCP-guided collection method, a guidewire was inserted into the stricture of the pancreatic or bile duct and the stricture was brushed 5-10 times with a brush catheter (Rapid Cytology Brush, Boston Scientific, Tokyo, Japan; BC-24Q Disposable Cytology Brush, Olympus, Tokyo, Japan; or Fusion Cytology Brush, Cook Medical, Winston-Salem, NC, USA) that was inserted with the ropeway method. After brushing, only the inner tube of the brush catheter was removed and bile or pancreatic juice was collected by aspiration in the pancreatic or bile duct proximal and distal to the stricture. Only the tip of the brush catheter was removed and, following immersion in physiological saline or a preservative solution (Cyto-rich ${ }^{\circledR}$ Red Preservative, Beckton-Dickinson, Franklin Lanes, NJ, USA), it was manually stirred and the pellet obtained by centrifugation (840 x g x $5 \mathrm{~min}$ ) was used to prepare the slide specimens. The bile or pancreatic fluid after brushing was also centrifuged in a similar manner and slide specimens were prepared.

EUS-FNA specimen collection was performed mainly using a 22G/25G puncture needle; $15-20$ strokes and 3-5 sessions were performed. The specimen was fixed in formalin and submitted for cytology. Rapid on-site evaluation (ROSE) was also performed in each case.

Patient grouping. The subjects were divided into two groups, one prior to 2006 (group A), when the ERCP-guided collection method was considered the first choice, and one from 2007 onwards (group B), after the introduction of EUS-FNA. We compared the results of cytology and histological diagnosis in the two groups and according to the lesion site (pancreatic head vs. body and tail) and assessed the incidence and nature of complications.

When the results of cytology or histological diagnosis were 'malignant' or 'suspected malignant' the results were considered to be cancer-positive. In addition, in cases where ERCP-guided bile and pancreatic duct cytology was performed, specimens of
Table I. Patient characteristics.

\begin{tabular}{lccc}
\hline Characteristics & Group A & Group B & P-value \\
\hline $\begin{array}{l}\text { No. of patients } \\
\text { Age, years }\end{array}$ & 101 & 162 & \\
mean (range) & $68.91(35-91)$ & $68.24(33-90)$ & 0.631 \\
Gender, male/female & $59 / 42$ & $94 / 68$ & 0.950 \\
Location of the & & & \\
pancreatic tumor & & $74(50)$ & \\
Head (presence of & $59(39)$ & & \\
obstructive jaundice) & & 88 & \\
Body and tail & 42 & & \\
Stage (JPS) & & 0.306 \\
1 & 0 & 3 & \\
2 & 0 & 9 & \\
3 & 8 & 54 & \\
$4 \mathrm{a}$ & 36 & 96 & \\
$4 \mathrm{~b}$ & 57 & & \\
\hline
\end{tabular}

JPS, Japan Pancreas Society.

either method that were positive were considered to be cancerpositive.

Statistical analysis. The JMP ${ }^{\circledR} 10$ software program (SAS Institute Inc., Cary, NC, USA) was used to perform the statistical analysis and a $\mathrm{P}<0.05$ was considered to indicate a statistically significant difference.

\section{Results}

Patient characteristics. The 263 PC cases that were diagnosed as unresectable included 101 cases in group A and 162 in group B. The differences between the groups regarding age, gender, tumor location, presence or absence of obstructive jaundice and stage are summarized in Table I.

Comparison of endoscopic cytology results between groups $A$ and $B$. ERCP-guided cytology was performed in all 101 patients in group A, with a cancer-positive rate of $60.4 \%$ (61/101). The 162 patients in group B included 92 patients in the ERCP-guided cytology 1st group and 70 patients in the EUS-FNA 1st group. The cancer-positive rate was $45.7 \%$ (42/92) in the ERCP-guided cytology 1st group and $94.3 \%$ (66/70) in the EUS-FNA 1st group. Consent to subsequently perform EUS-FNA was obtained from 22 of the 50 patients in the ERCP-guided cytology 1st group who were cancer-negative and the cancer-positive rate was $63.6 \%$ (14/22). Collectively, the cancer-positive rate was $60.4 \%$ $(61 / 101)$ in group A and $75.3 \%(122 / 162)$ in group B; the difference was statistically significant $(\mathrm{P}=0.01)$. Furthermore, when the 28 patients in group B who did not consent to EUS-FNA after ERCP were excluded, the cancer-positive rate in group B group was $91.0 \%(122 / 134)$, which was significantly higher compared to that in group A $(\mathrm{P}<0.001)$ (Fig. 1).

Endoscopic cytology results by location. The cancer-positive rate of the lesions located in the pancreatic head was $61.0 \%$ (36/59) in group A and 67.9\% (57/84) in group B; the difference 


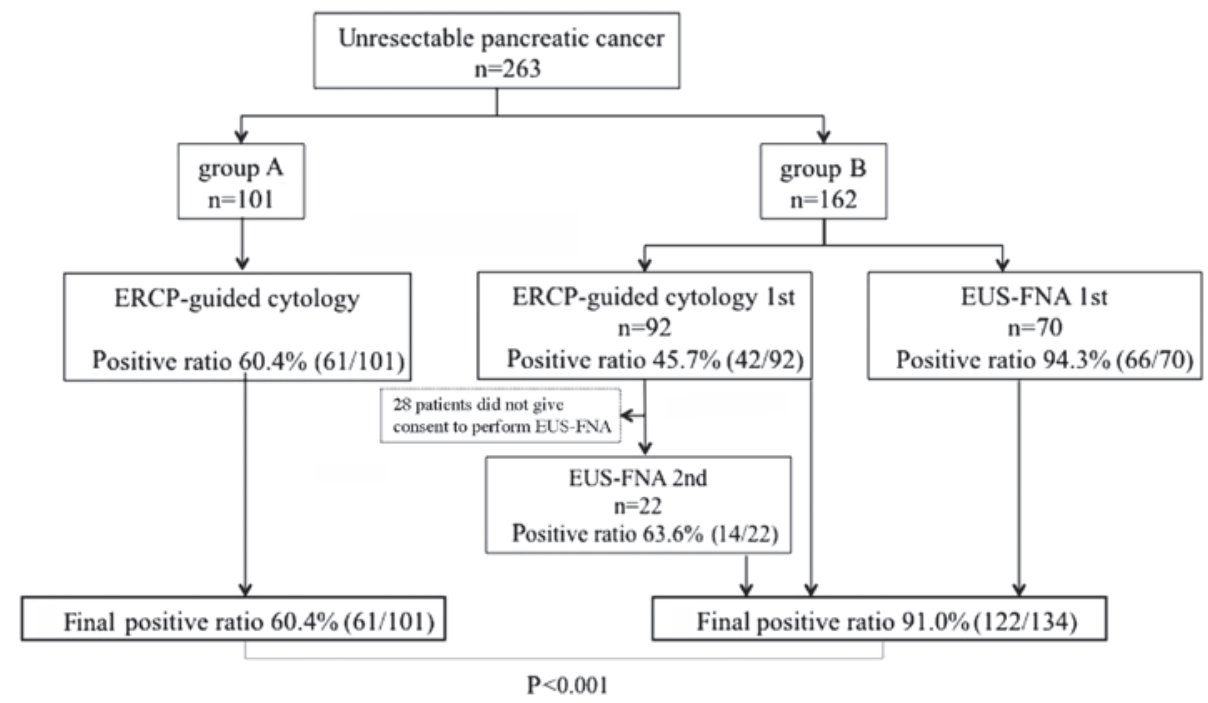

Figure 1. Endoscopic cytology results in patients with unresectable pancreatic cancer. ERCP, endoscopic retrograde cholangiopancreatography; EUS-FNA, endoscopic ultrasound-guided fine-needle aspiration.

Table II. Comparison of endoscopic cytology results according to the location of the pancreatic cancer between groups A and B.

\begin{tabular}{|c|c|c|c|c|}
\hline Location of pancreatic cancer & Group A & \multicolumn{2}{|c|}{ Group B } & P-value \\
\hline \multirow[t]{4}{*}{ Head } & $61.0 \%(36 / 59)$ & \multicolumn{2}{|c|}{$67.9 \%(57 / 84)$} & 0.134 \\
\hline & & ERCP 1st: & $48.5 \%(32 / 66$ & \\
\hline & & EUS-FNA 1st: & $88.9 \%(16 / 18)$ & \\
\hline & & EUS-FNA 2nd: & $60.0 \%(9 / 15)$ & \\
\hline \multirow[t]{4}{*}{ Body and tail } & $59.5 \%(25 / 42)$ & \multicolumn{2}{|c|}{$83.3 \%(65 / 78)$} & 0.005 \\
\hline & & ERCP 1st: & $38.5 \%(10 / 26)$ & \\
\hline & & EUS-FNA 1st: & $96.2 \%(50 / 52)$ & \\
\hline & & EUS-FNA 2nd: & $71.4 \%(5 / 7)$ & \\
\hline
\end{tabular}

ERCP, endoscopic retrograde cholangiopancreatography; EUS-FNA, endoscopic ultrasound-guided fine-needle aspiration.

was not statistically significant. The cancer-positive rate in the pancreatic body and tail was $59.5 \%(25 / 42)$ in group A and $83.3 \%(65 / 78)$ in group B and the difference was statistically significant $(\mathrm{P}=0.005)$. The cancer-positive rate of the EUS-FNA cases alone in group B was $75.7 \%(25 / 33)$ in the pancreatic head and $93.2 \%(55 / 59)$ in the pancreatic body and tail, with a statistically significant difference $(\mathrm{P}=0.017)$ (Table II).

Comparison of consistency and frequency of complications between the two groups. The incidence of complications was $4.95 \%(5 / 101)$ in group A and $3.09 \%(5 / 162)$ in group B $(\mathrm{P}=0.448)$. When the patients in group $\mathrm{B}$ who did not consent to EUS-FNA after ERCP were excluded, the incidence of complications in group B was $3.73 \%$ (5/134). In group A, the complications included PEP in 4 cases (mild in 3 and moderate in 1) and hemorrhage due to endoscopic sphincterotomy in 1 case; in group B, PEP was observed in 4 cases (mild in 3 and moderate in 1) and post-EUS-FNA pancreatitis (moderate) in 1 case. Hyperamylasemia developed in 9 cases in each group. All the complications improved with conservative treatment, without severe cases or fatalities (Table III).
Table III. Comparison of complication frequency between groups $\mathrm{A}$ and $\mathrm{B}$.

\begin{tabular}{lccc}
\hline $\begin{array}{l}\text { Complication } \\
\text { frequency }\end{array}$ & Group A & Group B & P-value \\
\hline Total & $4.95 \%(5 / 101)$ & $3.09 \%(5 / 162)$ & 0.4481 \\
Due to ERCP, & & & \\
Pancreatitis & $4^{\mathrm{a}}$ & $4^{\mathrm{b}}$ & \\
Hemorrhage & 1 & & \\
$\begin{array}{l}\text { Due to EUS-FNA, n } \\
\text { Pancreatitis }\end{array}$ & - & $1^{\mathrm{c}}$ & \\
\hline
\end{tabular}

${ }^{\mathrm{a}}$ Mild in 3 , moderate in 1 ; $^{\mathrm{b}}$ mild in 3 , moderate in 1 ; ${ }^{\mathrm{c}}$ moderate. ERCP, endoscopic retrograde cholangiopancreatography; EUS-FNA, endoscopic ultrasound-guided fine-needle aspiration.

\section{Discussion}

The PC diagnostic accuracy rate based on diagnostic imaging has improved as a result of advances in various diagnostic 
imaging equipment over the last few years; whether histological diagnosis is necessary when distant metastasis is present remains debatable. However, against a background of cases in which it is difficult to perform a differential diagnosis from inflammatory diseases and due to the recent advances in the multidisciplinary treatment of PC, it is crucial to obtain a histopathological diagnosis prior to treatment (5). Obtaining a pathological basis is crucial for undertaking chemotherapy and selecting the appropriate medication, particularly in cases with unresectable disease, in which a definitive pathological diagnosis is impossible.

Percutaneous and open biopsy have long been used to obtain PC pathology specimens; however, endoscopic non-invasive approaches have recently become mainstream. ERCP-guided specimen collection and EUS-FNA are currently preferred as endoscopic collection methods, although the ERCP-guided method has been used longer. ERCP-guided direct collection of specimens from the bile and pancreatic duct is considered a viable diagnostic method from the standpoint of ordinary PCs arising from the pancreatic duct epithelium; however, there is a wide variation (33-92\%) in the sensitivity of ERCP-guided specimen collection among institutions and its usefulness is not consistent (6-9).

Consequently, various modifications have been attempted to improve diagnostic performance. Pancreatic juice cytology, in which secretin was used to forcibly stimulate pancreatic juice secretion, was previously performed, with reported good results, i.e., 30-79\% (10). However, since 2004, when secretin became unavailable, reports on brush cytology, in which specimens are collected by forcibly brushing the pancreatic duct, have become more common. The diagnostic performance of brush cytology was reported to be $30-93 \%$, although there have been occasional reports of even more ingenious modifications (7). We previously reported that, when we collected pancreatic juice that had accumulated after brushing, the sensitivity improved from 62 to $73.7 \%$; the diagnostic performance was also improved (9). We hypothesized that these results were attributable to mechanically stripping the pancreatic duct epithelium, which enabled the collection of numerous fresh cells, thereby minimizing degeneration by pancreatic enzymes (11). Uehara et al (6) reported achieving $92 \%$ sensitivity by a method in which scraping was performed with a guidewire. Furthermore, Kimura et al (8) reported that it was possible to perform cytology several times by following ERCP with placement of an endoscopic naso-pancreatic drainage tube, with a sensitivity of $62 \%$.

After a report of EUS-FNA for PC by Vilmann et al (3) in 1992, EUS-FNA became widely adopted, primarily in Western countries. The results for diagnostic performance regarding pancreatic mass lesions revealed a sensitivity of $85-94 \%$, a specificity of $100 \%$ and an accuracy of $95 \%$, which were better compared to those with ERCP-guided cytology (12-14).

In the present study, the sensitivity of the ERCP-guided collection method was $60.4 \%$ in group A, $45.7 \%$ in group B, and $53.3 \%$ overall; thus, we obtained results consistent with those in previous reports. By contrast, the sensitivity of EUS-FNA in group B was $87.0 \%$ (EUS-FNA 1st, $94.3 \%$ and EUS-FNA 2nd, 63.6\%) and its diagnostic performance was superior to that of ERCP-guided cytology.

The fact that the cases in which ERCP-guided cytology was performed in group B were restricted to pancreatic head lesions that required EBS appears to have been a factor affecting the differences in the diagnostic performance of the ERCP-guided collection method between groups A and B. This difference may be due to the fact that the main pancreatic duct was obstructed in a number of the pancreatic head cancers and it was impossible to collect an adequate specimen by brushing.

The presence of more pancreatic head lesions in the EUS-FNA 2nd cases in group B may have been another factor responsible for the difference in sensitivity between EUS-FNA 1st and EUS-FNA 2nd cases in group B. A transduodenal approach is often used to perform EUS-FNA for pancreatic head lesions and puncture manoeuvres may prove difficult, as the tip of the scope bends sharply as a result of the endoscope angle. Therefore, diagnostic performance appears to be reduced by a technical problem $(15,16)$. Haba et al $(17)$ also reported poorer diagnostic accuracy for pancreatic head lesions compared to body and tail lesions. There was also a report according to which a $25 \mathrm{G}$ needle that makes puncture manoeuvres easier compared to conventional needles was recently developed and the specimen collection rate and diagnostic performance for pancreatic head lesions have been improved (18); however, additional testing is required. ROSE was also reported to improve diagnostic accuracy (19), contribute to increasing diagnostic performance, reducing the number of punctures and reducing the complication rate (20).

The incidence of complications attributable to ERCP is considered to be high for an endoscopic procedure and the incidence of PEP, in particular, was reported to be 2-9\% (21-23). By contrast, the incidence of complications in patients who undergo EUS-FNA was reported to be $<2 \%$, making EUS-FNA a relatively safe procedure $(24,25)$. In the present study, the overall incidence of PEP was $4 \%$, but the complications of EUS-FNA included only one case of pancreatitis (incidence rate of $1 \%$ ). Tumor seeding as a result of the puncture is a rare complication of EUS-FNA; to date, a total of 4 cases have been reported, i.e., a case of seeding of the abdominal cavity by a mucinous cystic tumor of the pancreas (4), a case of pancreatic metastasis by a malignant melanoma (26), seeding of the puncture tract by a pancreatic tail cancer (27) and seeding of a metastatic mediastinal lymph node after a transesophageal puncture (28). However, Ikezawa et al (29) reported that EUS-FNA of the pancreas does not increase the risk of seeding. It may be possible to reduce the problem of cancer seeding by EUS-FNA by excluding special cases, such as mucinous cystic tumors of the pancreas. In addition, a study conducted by Matsumoto et al (30) on a group of PC patients who were treated by chemotherapy, reported that there was no difference in the incidence rates of peritonitis carcinomatosa or ascites between patients who had undergone EUS-FNA and those who had not. Therefore, we do not consider EUS-FNA as disadvantageous, at least for patients with unresectable PC who are candidates for chemotherapy.

Certain recent studies reported that ERCP and EUS-FNA performed on the same day may be more efficient and cost-effective $(31,32)$. Those reports taken together with the results of the present study suggest that it may be more efficient to perform EUS-FNA on the same day as EBS in unresectable PC cases that are complicated by obstructive jaundice.

In conclusion, there was a significant difference in the endoscopic cytological diagnosis cancer-positive rate in patients with unresectable PC prior to and after the introduction of 
EUS-FNA, with the results improving after the introduction of EUS-FNA. In addition, although the difference in the complication rate prior to and after the introduction of EUS-FNA was not significant, a number of the complications were attributable to the ERCP procedure.

\section{References}

1. Lee JG and Leung J: Tissue sampling at ERCP in suspected pancreatic cancer. Gastrointest Endosc Clin N Am 8: 221-235, 1998.

2. Howell DA, Parsons WG, Jones MA, Bosco JJ and Hanson BL: Complete tissue sampling of biliary strictures at ERCP using a new device. Gastrointest Endosc 43: 498-502, 1996.

3. Vilmann P, Jacobsen GK, Henriksen FW and Hancke S: Endoscopic ultrasonography with guided fine needle aspiration biopsy in pancreatic disease. Gastrointest Endosc 38: 172-173, 1992.

4. Hirooka Y, Goto H, ltoh A, et al: Case of intraductal papillary mucinous tumor in which endosonography-guided fine-needle aspiration biopsy caused dissemination. J Gastroenterol Hepatol 18: 1323-1324, 2003.

5. Kichise J, Suzuki E, Hirokawa S, Kitamura H and Nakashima F: Significance of pathological examination in biliary tract and pancreatic cancer. J Biliary Tract Pancreas 31: 809-813, 2010 (In Japanese).

6. Uehara H, Tatsunami K, Masuda E, et al: Scraping cytology with a guidewire for pancreatic-ductal strictures. Gastrointest Endosc 70: 52-59, 2009

7. Arizumi T, Tada M, Togawa O, et al: Efficacy of combinatorial diagnosis of pancreatic cancer; Combination of variety of diagnostic modalities and specimen collecting method. Gastroenterology 128 (Suppl 2): A470, 2005.

8. Kimura K, Furukawa Y, Yamasaki S, et al: A study of the usefulness of pancreatic juice cytology obtained via an endoscopic nasal pancreatic drainage (ENPD) tube. Jpn J Gastroenterol 108: 928-936, 2011 (In Japanese).

9. Okabe Y, Naito Y, Kawahara A, et al: The Management of endoscopic transpapillary cytology for the pancreatic cancer. J Biliary Tract Pancreas 27: 157-161, 2006 (In Japanese).

10. Nakaizumi A, Tatsuta $\mathrm{M}$, Uehara $\mathrm{H}$, et al: Cytologic examination of pure pancreatic juice in the diagnosis of pancreatic carcinoma. The endoscopic retrograde intraductal catheter aspiration cytologic technique. Cancer 70: 2610-2614, 1992.

11. Naito Y, Okabe Y, Kawahara A, Taira T, Kusano H and Kage M: Study on the cytology of the pancreatic duct by different sampling. Jpn Soc Clin Cytol 46: 7-11, 2007 (In Japanese).

12. Yamao K, Sawaki A, Mizuno N, Shimizu Y, Yatabe Y and Koshikawa T: Endoscopic ultrasound-guided fine-needle aspiration biopsy(EUS-FNAB): past, present, and future. J Gastroenterol 40: 1013-1023, 2005.

13. Fisher L, Segarajasingam DS, Stewart C, Deboer WB and Yusoff F: Endoscopic ultrasound guided fine needle aspiration of solid pancreatic lesions: Performance and outcomes. J Gastroenterol Hepatol 24: 90-96, 2009.

14. Hewitt MJ, McPhail MJ, Possamai L, Dhar A, Vlavianos P and Monahan KJ: EUS-guided FNA for diagnosis of solid pancreatic neoplasma: a meta-analysis. Gastrointest Endosc 75: 319-331, 2012

15. Larghi A, Verna EC, Stavropoulos SN, Rotterdam H, Lightdale CJ and Stevens PD: EUS-guided trucut needle biopsies in patients with solid pancreatic masses: a prospective study. Gastrointest Endosc 59: 185-190, 2004.

16. Itoi $\mathrm{T}$, Tsuchiya $\mathrm{T}$, Itokawa $\mathrm{F}$, Sofuni $\mathrm{A}$, Kurihara $\mathrm{T}$, Tsuji $\mathrm{S}$ and Ikeuchi N: Histological diagnosis by EUS-guided fine-needle aspiration biopsy in pancreatic solid masses without on-site cytopathologist: a single-center experience. Dig Endosc 23 (Suppl 1): 34-38, 2011.
17. Haba S, Yamao K, Bhatia V, et al: Diagnostic ability and factors affecting accuracy of endoscopic ultrasound-guided fine needle aspiration for pancreatic solid lesions: Japanese large single center experience. J Gastroenterol 48: 973-981, 2013.

18. Sakamoto H, Kitano M, Komaki T, et al: Prospective comparative study of the EUS guided 25-gauge FNA needle with the 19-gauge Trucut needle and 22-gauge FNA needle in patients with solid pancreatic masses. J Gastroenterol Hepatol 24: 384-390, 2009

19. Hikichi T, lrisawa A, Bhutani MS, et al: Endoscopic ultrasound-guided fine-needle aspiration of solid pancreatic masses with rapid on-site cytological evaluation by endosonographers without attendance of cytopathologists. J Gastroenterol 44: 322-328, 2009.

20. Iglesias-Garcia J, Dominguez-Munoz JE, Abdulkader I, LarinoNoia J, Eugenyeva E, Lozano-Leon A and Forteza-Vila J: Influence of on-site cytopathology evaluation on the diagnostic accuracy of endoscopic ultrasound-guided dine needle aspiration (EUS-FNA) of solid pancreatic masses. Am J Gastroenterol 106 1705-1710, 2011.

21. Freeman ML, DiSario JA, Nelson DB, et al: Risk factors for post-ERCP pancreatitis: a prospective, multicenter study. Gastrointest Endosc 54: 425-434, 2001.

22. Wang P, Li ZS, Liu F, et al: Risk factors for ERCP related complications: a prospective multicenter study. Am J Gastroenterol 104: 31-40, 2009.

23. Williams EJ, Taylor S, Fairclough P, et al: Risk factors for complication following ERCP; results of a large-scale, prospective multicenter study. Endoscopy 39: 793-801, 2007.

24. Yamao K: Complications of endoscopic ultrasound guided fine-needle aspiration biopsy (EUS-FNAB) for pancreatic lesions. J Gastroenterol 40: 921-923, 2005.

25. Polkowski M, Larghi A, Weynand B, Boustlère C, Glovannini B, Pujol B and Dumonceau JM; European Society of Gastrointestinal Endoscopy (ESGE): Learning, techniques, and complications of endoscopic ultrasound (EUS)-guided sampling in gastroenterology: European Society of Gastrointestinal Endoscopy (ESGE) Technical Guideline. Endoscopy 44: 190-206, 2012.

26. Shah JN, Fraker D, Guerry D, Feldman M and Kochman ML: Melanoma seeding of an EUS-guided fine needle track. Gastrointest Endosc 59: 923-924, 2004.

27. Paquin SC, Gariépy G, Lepanto L, et al: A first report tumor seeding because of EUS-guided FNA of a pancreatic adenocarcinoma. Gastrointest Endosc 61: 610-611, 2005.

28. Doi S, Yasuda I, lwashita T, et al: Needle tract implantation on the esophageal wall after EUS-guided FNA of metastatic mediastinal lymphadenopathy. Gastrointest Endosc 67: 988-990, 2008.

29. Ikezawa K, Uehara H, Sakai A, et al: Risk of peritoneal carcinomatosis by endoscopic ultrasound-guided fine needle aspiration for pancreatic cancer. J Gastroenterol 48: 966-972, 2013.

30. Matsumoto K, Yamao K, Ohashi K, et al: The clinical utility of EUS-guided fine-needle aspiration (EUS-FNA) for pancreatic lesions. J Jpn Pancreas Soc 17: 485-491, 2002 (In Japanese).

31. Aslanian HR, Estrada JD, Rossi F, Dziura J, Jamidar PA and Siddiqui UD: Endoscopic ultrasound and endoscopic retrograde cholangiopancreatography for obstructing pancreas head masses: combined or separate procedures? J Clin Gastroenterol 45: 711-713, 2011.

32. Ross WA, Wasan SM, Evans DB, et al: Combined EUS with FNA and ERCP for the evaluation of patients with obstructive jaundice from presumed pancreatic malignancy. Gastrointest Endosc 68: 461-466, 2008 